\title{
Propagating brightenings in small loop-like structures in the quiet-Sun corona: Observations from Solar Orbiter/EUI*
}

\author{
Sudip Mandal ${ }^{1}$, Hardi Peter ${ }^{1}$, Lakshmi Pradeep Chitta ${ }^{1}$, Sami K. Solanki ${ }^{1,2}$, Regina Aznar Cuadrado ${ }^{1}$, Luca Teriaca ${ }^{1}$, \\ Udo Schühle $^{1}$, David Berghmans ${ }^{3}$, and Frédéric Auchère ${ }^{4}$
}

\author{
1 Max Planck Institute for Solar System Research, Justus-von-Liebig-Weg 3, 37077 Göttingen, Germany \\ e-mail: smandal.solar@gmail.com \\ 2 School of Space Research, Kyung Hee University, Yongin, Gyeonggi 446-701, Republic of Korea \\ 3 Royal Observatory of Belgium, Ringlaan -3- Av. Circulaire, 1180 Brussels, Belgium \\ 4 Institut d'Astrophysique Spatiale, CNRS, Univ. Paris-Sud, Université Paris-Saclay, Bât. 121, 91405 Orsay, France
}

Received 17 August 2021 / Accepted 15 November 2021

\begin{abstract}
Brightenings observed in solar extreme-ultraviolet images are generally interpreted as signatures of micro- or nanoflares occurring in the transition region or at coronal temperatures. Recent observations with the Extreme Ultraviolet Imager (EUI) on board Solar Orbiter have revealed the smallest of such brightenings (called campfires) in the quiet-Sun corona. Analyzing EUI 174 Å data obtained at a resolution of about $400 \mathrm{~km}$ on the Sun with a cadence of $5 \mathrm{~s}$ on 30 May 2020, we report here a number of cases in which these campfires exhibit propagating signatures along their apparently small (3-5 Mm) loop-like structures. The measured propagation speeds are generally between $25 \mathrm{~km} \mathrm{~s}^{-1}$ and $60 \mathrm{~km} \mathrm{~s}^{-1}$. If the loop plasma is assumed to be at a million Kelvin, these apparent motions would be slower than the local sound speed. Furthermore, these brightenings exhibit nontrivial propagation characteristics such as bifurcation, merging, reflection, and repeated plasma ejections. We suggest that these features are manifestations of the internal dynamics of these small-scale magnetic structures and could provide important insights into the dynamic response ( $\sim 40 \mathrm{~s})$ of the loop plasma to the heating events and also into the locations of the heating events themselves.
\end{abstract}

Key words. Sun: corona - Sun: magnetic fields

\section{Introduction}

Localized brightenings are commonly detected using ultraviolet (UV) or extreme-ultraviolet (EUV) imaging and spectroscopic diagnostics of the solar chromosphere through the corona. These brightenings are generally compact and dynamic and are thought to be driven by magnetic reconnection. Depending upon their imaging and spectroscopic signatures (including energetics), these bright features are often referred to in the literature as bright points (Madjarska et al. 2003), explosive events (Brueckner \& Bartoe 1983; Dere et al. 1989; Innes et al. 1997), blinkers (Harrison 1997), or UV bursts (Peter et al. 2014). For a comprehensive review of this subject, see Young et al. (2018). Multi-instrument observations further revealed that these bright features appear everywhere on the solar disk, that is, from quiet-Sun areas to active region neighborhoods (Krucker et al. 1997; Berghmans et al. 1998; Berghmans \& Clette 1999; Aschwanden et al. 2000a; Tiwari et al. 2019). Because they are nearly ubiquitous, these brightenings were initially thought to be potential candidates for coronal heating (Hudson 1991). However, further investigations indicate that significantly more ( $\sim 100$ times) of these events would be required in order to account for the necessary energy budget (Aschwanden et al. 2000b; Chitta et al. 2021a).

\footnotetext{
* Movies associated to Figs 2-5, A.1, and B.1 are available at https://www . aanda.org
}

Recent observations from the Extreme Ultraviolet Imager (EUI; Rochus et al. 2020) on board Solar Orbiter (Müller et al. 2020) have uncovered localized brightenings (called campfires) down to sizes as small as $0.08 \mathrm{Mm}^{2}$ (Berghmans et al. 2021). These are the smallest such events observed in the quiet-Sun corona to date. Although most of these campfire events observed with EUI are also detected with the Atmospheric Imaging Assembly on board the Solar Dynamics Observatory (AIA/SDO; Lemen et al. 2012), their AIA counterparts appear to be rather faint and fuzzy. This is mostly due to the coarser spatial and temporal resolutions of AIA. In an effort to explain the physical origin of these brightenings, Berghmans et al. (2021) proposed a scenario in which the apex of a low-lying coronal or transition region loop (Zhukov et al. 2021) is heated by (component) magnetic reconnection, and the heated plasma subsequently appears as a localized brightening. This interpretation was substantiated by Chen et al. (2021), who found similar campfire-like events in a 3D radiation magnetohydrodynamic (MHD) simulation. These authors have also demonstrated that different magnetic configurations are possible that might trigger these campfires (e.g., forking field lines or crossing field lines).

Reports of subarcsecond brightenings using high-resolution data from previous missions such as IRIS (De Pontieu et al. 2014) and Hi-C (Kobayashi et al. 2014; Rachmeler et al. 2019) have mostly been limited to regions that are either close to or lie within an active region (Winebarger et al. 2013; Tian et al. 2014; Peter et al. 2014; Alpert et al. 2016; Tiwari et al. 2019). 




Fig. 1. Internal dynamics in small loop-like features. Panel a: time-averaged image of the HRI $\mathrm{EUv}_{\mathrm{v}}$ observation on 30 May 2020 . White boxes outline the locations of the six events studied here. Panels $b$ and $d$ : snapshots from events 1 and 2 and from event 4 , respectively. In each of these snapshots, the curved blue box marks the extent of the artificial slit that is used to derive the space-time map, and the arrow points to the instantaneous position of the localized brightening in that frame. The elapsed time for each frame is given in seconds as measured from 14:54:00 UT, i.e., the start time of the observation.

Tian et al. (2014) found subarcsecond brightenings in IRIS data within sunspot penumbrae that have speeds between $10-40 \mathrm{~km} \mathrm{~s}^{-1}$. On the other hand, analyzing $\mathrm{Hi}-\mathrm{C}$ data, Winebarger et al. (2013) reported localized plasma flows at high speeds (between 90-290 $\mathrm{km} \mathrm{s}^{-1}$ ) within small loops that are embedded inside a moss region. Recently, a statistical study of EUV bursts in quiet-Sun regions has been presented in Chitta et al. (2021a). The detection of these burst-like features was primarily constrained by the coarser spatial resolution of the AIA data (about $900 \mathrm{~km}$ ) used in that study. Furthermore, this limitation also meant that the internal dynamics of these EUV bursts could not be studied with AIA.

In this Letter, we analyze the EUI data taken during the commissioning phase of the Solar Orbiter mission (Müller et al. 2020) and present a number of localized brightening events in the quiet-Sun region that exhibit systematic propagation signatures along their host loop-like structures. The EUI imaging data facilitate studying the dynamical nature of these brightenings in greater detail because their spatial resolution is higher (about $400 \mathrm{~km})$ and the image cadence is finer $(5 \mathrm{~s})$ that for AIA data. We describe the data in Sect. 2, and Sect. 3 outlines the results. Finally, we conclude by summarizing our results in Sect. 4.

\section{Data}

We use EUV imaging data ${ }^{1}$ from the High Resolution Imager (HRI) of the Extreme Ultraviolet Imager (EUI; Rochus et al. 2020) on board Solar Orbiter. These images were taken on 30 May 2020 with the $\mathrm{HRI}_{\mathrm{EUV}}$ telescope, which images a wave-

\footnotetext{
1 We used level 2 data (L2), which can be accessed via https:// wwwbis.sidc.be/EUI/data/releases/202107_release_3.0/.

Information about the data processing can be found in the release notes. DOI: https://doi .org/10.24414/k1xz-ae04.
}

length band around $174 \AA$. This band captures the dynamics of the solar plasma with temperatures between $\log T[\mathrm{~K}]=5.3$ and 6.3 (i.e., transition region to cooler corona) with a peak near $1 \mathrm{MK}$. We align the individual images using the ssw routine fg_rigidalign.pro, which removes the effect of jitter in the data by means of cross-correlations. The image cadence is $5 \mathrm{~s}$, and a total of 50 frames were recorded. The pixel scale is $0.492^{\prime \prime}$ in each direction. At the time of this observation, Solar Orbiter was located at a distance of about 0.556 astronomical units from the Sun. Hence, each pixel in these images corresponds to a distance of $198 \mathrm{~km}$ on the solar surface at disk center. Further details about this observational campaign can be found in Berghmans et al. (2021).

\section{Results}

\subsection{General characteristics}

Figure 1a shows the full $406 \times 406 \mathrm{Mm}^{2}$ field of view (FOV) of the analyzed observation. It primarily encompasses a quietSun region near the disk center (as seen from the vantage point of Solar Orbiter). The overplotted white boxes outline the locations of the six events that we analyze here. Except for one case (event 3), all events are located within relatively quiescent regions in the FOV. A feature that is common to all these events is the systematic propagation of a localized brightening along the loop-like host structure (see Figs. 1b-d and online animations). These propagating brightenings are mostly between 5 to 20 pixels (i.e., between 0.2 to $0.8 \mathrm{Mm}^{2}$ ) in size, and their appearance is comparable to the moderate-size campfires reported in Berghmans et al. (2021). We also note that the structures hosting these brightenings appear to be small loop-like configurations with footpoint separations of 2 to $4 \mathrm{Mm}$. 



Fig. 2. Bifurcation scenario 1 (event 1). Panel a: context image for this event along with the artificial slit (curved blue box) that we used to generate the space-time $(X-T)$ map (panel $b$ ). The background-subtracted version of this map is shown in panel $c$. The label 'start' ('end') in panel $a$ refers to the origin (end point) of the $y$-axis of the $X-T$ map. The inclined dash-dotted green and orange lines in panel $c$ represent the slopes of the individual ridges we used to estimate the speed. The dash-dotted orange lines encapsulate the inverse-v shape that indicates a reflection at the loop footpoint. Panels $d-f$ : light curves that we extracted from the three square boxes B1, B2, and, B3 as shown in panel a. The center points of these boxes are highlighted as horizontal lines in panel-b. For more details, see Sect. 3.2. (An animated version of this figure is available online.)

In order to highlight the overall temporal characteristics of these events, snapshots from three of these events are presented in Figs. 1b (event 1), c (event 2), and d (event 4). These plots show that the analyzed compact brightenings appear to propagate, although the propagation characteristics in each of these events are distinctly different. For example, the brightening in event 1 first appears near the middle of the structure, whereas in the other two examples, it appears close to the footpoints. The spatial extent (and shape) of these features is also noteworthy. In event 1, the brightening is rather compact (point-like), whereas in event 4 , it is quite elongated (loop-like). Similar shape distinctions were also reported in Tiwari et al. (2019) and Panesar et al. (2021).

To explore these aspects is more detail, we present in-depth descriptions of each of these events in the following sections. We present four of these events in the main paper, and the remaining two are discussed in the appendix.

\subsection{Event 1: Bifurcation scenario 1}

This particular event occured close to the upper edge of the observation FOV (see Fig. 1a). The host, in this case, appears to be a small loop whose plane-of-sky length is approximately $4 \mathrm{Mm}$. To capture the propagation signatures, we first construct a space-time $(X-T)$ map by placing an artificial slit that traced the propagation path of the observed brightening. The curved blue box in Fig. 2a highlights this artificial slit (the $X$ coordinate runs from the top left end of the curved slit to its bottom). The corresponding $X-T$ map is shown in Fig. 2b. Additionally, to enhance the contrast of this map, we detrend (and normalize) the intensity time series at every spatial location using a $150 \mathrm{~s}(30$ frames) running average. Figure 1c shows this enhanced $X-T$ map. This map shows that the localized brightening first appears near the middle of the loop at $t=10 \mathrm{~s}$. At $t=35 \mathrm{~s}$, it bifurcates into two smaller brightenings. These two features then start moving in opposite directions (i.e., toward the apparent footpoints of the host structure).

In the $X-T$ map, this diverging propagation is seen as two oppositely slanted ridges between $t=35 \mathrm{~s}$ and $t=80 \mathrm{~s}$. The propagation speeds (projected or plane-of-sky speeds) are measured as $22 \mathrm{~km} \mathrm{~s}^{-1}$ and $53 \mathrm{~km} \mathrm{~s}^{-1}$. Considering that the loop plasma is at one million Kelvin (which is reasonable given that the $174 \AA$ response function peaks at $\approx 1 \mathrm{MK}$ ), these speeds are well below the local sound speeds (which is $\approx 150 \mathrm{~km} \mathrm{~s}^{-1}$ ). One of the propagating features (the one between B2 and B1; Fig. 2a) moves from one end of its path to the other, after which it starts to return along its original path (highlighted with white arrows in Fig. 1b). This returning feature moves with a speed of $48 \mathrm{~km} \mathrm{~s}^{-1}$ toward the apparent loop apex (i.e., toward B2). It is this back and forth motion that produces the inverted v-shape (reflectionlike) signature in the $X-T$ map (this shape is outlined by the two orange lines in Fig. 2c). Interestingly, no such return feature is observed for the other brightening that moved toward the other footpoint (i.e., B3). Later, at $t=170 \mathrm{~s}$, a new brightening appears near the loop apex. It covers a significant portion of the loop (evident through the vertical extent of the feature in the $X-T$ map). In comparison, the initial brightening around $t=35 \mathrm{~s}$ is more localized before it propagates. Moreover, the second brightening (at $t=170 \mathrm{~s}$ ) showed no clear sign of propagation (as we thought from visual inspection) during its entire lifetime of $\approx 80 \mathrm{~s}$.

In order to further investigate the temporal evolution of the observed phenomenon, we now analyze light curves from three different locations within this loop-like structure. Three boxes, each $3 \times 3$ pixels in size, mark the locations (Fig. $2 a$ ) from which we extracted the light curves: B1 near the right footpoint, B2 at the apex, and B3 near the other footpoint. The extracted light curves are presented in panels $2 \mathrm{~d}$-e. There are several interesting features to highlight here. The light curves from the two opposite ends of the loop, that is, from B1 and B3, show rising trends well before the propagating brightenings from $\mathrm{B} 2$ actually reached them. This suggests the following scenario: although the main reconnection takes place near the apparent loop apex, the footpoints become excited simultaneously, which is similar to the near-simultaneous footpoint brightenings observed in active region core loops (see Chitta et al. 2020). It is also possible, however, that (undetected) fainter propagating brightenings from 

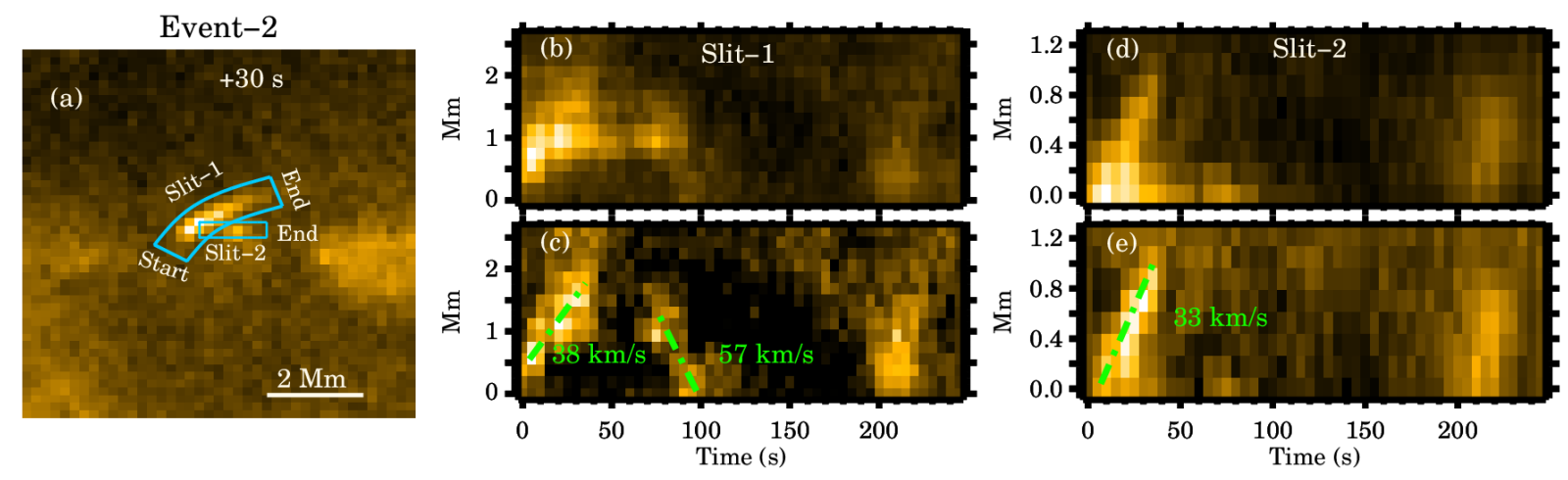

Fig. 3. Overview of bifurcation scenario 2 (event 2). The layout is similar as in Figs. 2a-c. Panel a: context image. The curved blue boxes mark the locations of the artificial slits (slit 1 and slit 2). Panels $b$ and $c$ : original and background-subtracted space-time maps for slit 1 . Panels $d$ and $e$ : the same but, for slit 2. The dashed green lines indicate the slopes of the ridges we used to estimate the speed. See Sect. 3.3 for details. An animated version of this figure is available online.

B2 have excited the footpoints B1 and B3. In this scenario, the observed delay between the first peak in B1 (B3) and that in B2 occurs due to the finite propagation time that the brightening at B2 takes to reach to that footpoint. Additionally, at the time of the second peak in $\mathrm{B} 2$ (associated with reflection from B1), a weak second peak (at $t=115 \mathrm{~s}$ ) occurs in B3. This could mean that there exists a faint 'reflected' brightening from this footpoint, too. However, this feature is rather unrecognizable in the original $X-T$ map (Fig. 2b) and also in the event movie. The last peak (at $t=170 \mathrm{~s}$ ) in all three light curves is basically due to the new extended brightening that appears again near the middle of the loop.

\subsection{Event 2: Bifurcation scenario 2}

In event 2 (Fig. 3a), the brightening first appears near the left footpoint of its host loop. The plane-of-sky extent of this loop is approximately $2 \mathrm{Mm}$. As soon as the brightening starts to move toward the right footpoint, a small bright feature detaches from this initial brightening and propagates away from the loop. Therefore, this is similar to the bifurcation scenario we found in event 1 . With time, this small bifurcated feature fades away rather quickly after traveling a short distance away for the loop, whereas the initial brightening could be traced to the other footpoint before it is reflects back to its starting location. Slits 1 and 2 in Fig. 3a outline the propagation paths of the initial and the detached brightenings, respectively. The space-time maps created using these two slits are shown in Figs. 3b-e (and are created in the same way as described in Sect. 3.2). In these two maps, we again find the propagation speeds to be subsonic (ranging between $33 \mathrm{~km} \mathrm{~s}^{-1}$ and $57 \mathrm{~km} \mathrm{~s}^{-1}$ ). Last, we also note that the bright feature seen at $t=205 \mathrm{~s}$ in Fig. 3b (also in Fig. 3d) is due to an extended brightening that appears momentarily and has no clearly detectable propagation characteristics.

\subsection{Event 3: Merger}

While events 1 and 2 display apparent bifurcations of the intensity propagation, event 3 (Fig. 4a) exhibits a different propagation signature: Here two separate brightenings come together and become a single entity, they merge. The initial two brightenings, one each at the two footpoints of the host structure, were already present in the first frame of this observation. Over time, the brightening at the right footpoint (labeled 'start' in Fig. 4a) moves toward the other footpoint (labeled 'end'). Interestingly,

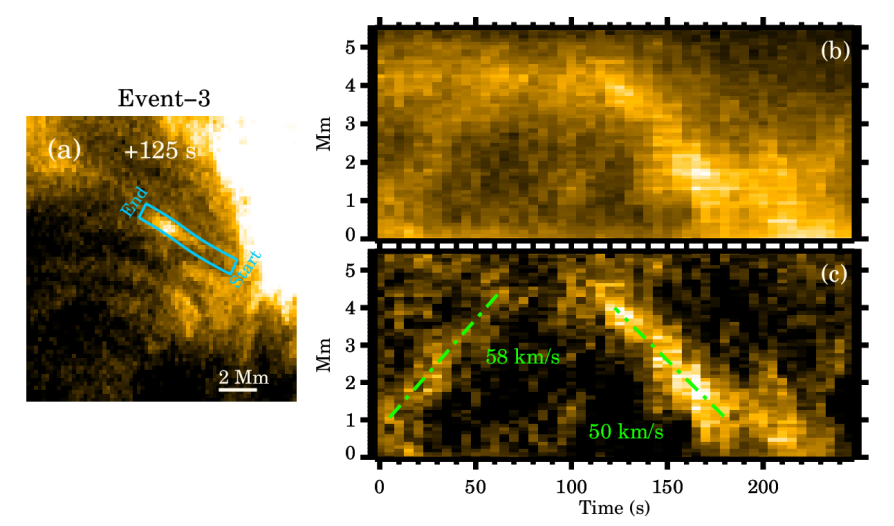

Fig. 4. Merger-type event (event 3). The layout is the same as in Figs. 2a-c. For details, see Sect. 3.4. An animated version of this figure is available online.

throughout this time, the other brightening at the left footpoint remains stationary. In the $X-T$ map (Fig. 4b), the stationary brightening appears as a horizontal bright ridge near the top of the map, wherein the slanted ridge that appears between $t=5 \mathrm{~s}$ and $t=80 \mathrm{~s}$ is due to the upward-moving brightening. Subsequently, these two brightenings meet near the left footpoint, and then the combined structure propagates back toward the right footpoint. As a result of this, we find a downward slanted bright streak in the $X-T$ map between $t=100 \mathrm{~s}$ and $t=170 \mathrm{~s}$. At this point, the intensity of the return brightening is higher than that of the original outward directed one. The speeds of the outward and return propagation are measured as $58 \mathrm{~km} \mathrm{~s}^{-1}$ and $50 \mathrm{~km} \mathrm{~s}^{-1}$, respectively (Fig. 4c). The location of this event is not strictly within a quiet-Sun patch, but rather close to a region with enhanced emission.

\subsection{Event 4: Multiple ejections}

Event 4 (Fig. 5a) is somewhat different compared to the events we have discussed so far. In this case, two successive brightenings propagate from one end of the structure to the other (Fig. 5b). These two ejections occur $50 \mathrm{~s}$ apart, and their speeds are estimated as $38 \mathrm{~km} \mathrm{~s}^{-1}$ and $26 \mathrm{~km} \mathrm{~s}^{-1}$, respectively (Fig. 5c). A distinct propagation characteristics is that the second ridge in the $X-T$ map becomes flatter at later times (around $t=180 \mathrm{~s}$ ). There is, however, no such clear trend in the first ridge, indicating that the second brightening might have reached farther 


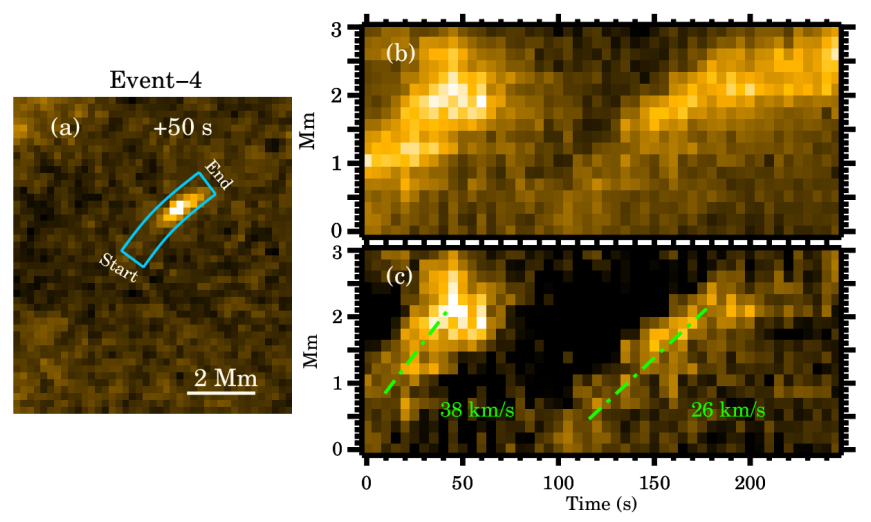

Fig. 5. Multiple-ejection-type event (event 4). The layout is the same as in Figs. 2a-c. For details, see Sect. 3.5. An animated version of this figure is available online.

toward this footpoint, and due to the geometry of a nearly vertical loop, the brightening appears to be decelerating. The lack of an apparent acceleration at the beginning of its journey suggests that either the brightening started higher up in the loop, or that it started with a high initial speed and decelerated slowly. Furthermore, the intensity of the second brightening increases gradually while it moves along the loop (Fig. 5b). This effect is rather weakly visible in the first brightening and can be attributed to the fact that the brightening already moved partway along the slit even before the time series began.

\section{Discussion and conclusion}

In this Letter, we presented evidence of propagating brightenings in small loop-like structures as observed in time series of EUI high-resolution images in the $174 \AA$ channel. The identification of the shapes of the loop we investigated, in particular, the location of their apex and footpoints, is based solely on the propagation characteristics of the brightenings. For a more reliable identification, the underlying magnetic field information at a comparably high spatial resolution would be required. While these were not available for these data from the early mission phase, co-observations with the Polarimetric and Helioseismic Imager on board Solar Orbiter (SO/PHI, Solanki et al. 2020) will become available for future studies.

By analyzing multiple of the propagating coronal brightenings in campfire loop-like events, we find that

1. the initial brightening appears either near the middle (apex) of the loop or near to one of the loop footpoints,

2. except for one case, the apparent propagation speeds lie between $25 \mathrm{~km} \mathrm{~s}^{-1}$ and $60 \mathrm{~km} \mathrm{~s}^{-1}$, which is below the local sound speed,

3. these brightenings exhibit nontrivial propagation characteristics such as bifurcation, back and forth motion, an apparent merging of brightenings, and repeated ejections.

These features are distinctly different from the bi- and unidirectional plasma jets that have recently been reported by Chitta et al. (2021b). Using an EUI data set with an image cadence of $2 \mathrm{~s}$, Chitta et al. (2021b) found compact jets at high speeds between $100 \mathrm{~km} \mathrm{~s}^{-1}$ and $150 \mathrm{~km} \mathrm{~s}^{-1}$. These are not only significantly faster than the propagating brightenings we report here, but are also different in morphology and propagation characteristics. This indicates a different driving mechanism.

As discussed earlier in Sect. 3.1, the brightenings whose propagation characteristics we analyzed here are mostly campfire-like features (Berghmans et al. 2021). Although campfires are the smallest such events observed in the quiet-Sun corona so far, it is not yet clear whether they are (physically) different from some of the already known brightening phenomena such as coronal bright points or the subarcsecond transition region brightenings. To distinguish these scenarios, simultaneous spectroscopic data are required (e.g., from SPICE, Spice Consortium 2020), which are not available for the observations we analyzed. Nevertheless, based on their rapid evolution, it is quite evident that these brightenings are likely products of magnetic reconnection. Different possible topologies might originate from the underlying magnetic fields that are prone to reconnection (Zhang et al. 2012; Innes et al. 2015; Galsgaard et al. 2017; Chitta et al. 2017; Madjarska 2019). Nonetheless, we can use the propagation characteristics of the observed brightenings to infer the underlying magnetic topology. For example, the bifurcation scenario we find in event 1 could be explained by a reconnection between two crossing field lines. The bifurcation (and subsequent propagation) seen in event 2 might be a signature of component reconnection in tangled field lines (e.g., similar to the one shown in Fig. 4d of Chen et al. 2021). Due to the lack of information about the underlying photospheric magnetic field at matching spatial resolution (the footpoints of these campfire loops are not resolved with SDO/HMI), we cannot confirm or disprove these different scenarios at this point. Co-observations with $\mathrm{SO} / \mathrm{PHI}$ that will be available in future observations will provide more insight into this question.

In two of our events (events 1 and 3), we have found brightenings that after traveling from one end of the host (loop-like) structure to the other, were reflected back. These cases appear similar to gentle chromospheric evaporation, in which nonthermal electrons from the site of a flare meet the chromospheric material at the footpoints of the loop along which they propagate and produce evaporated material upflow at a speed of tens of $\mathrm{km} \mathrm{s}^{-1}$ (Fisher et al. 1985; Milligan et al. 2006). Such a scenario has also been shown to hold not only for larger flares, but also for microflare-type outbursts (Brosius \& Holman 2009). However, certain features in our events appear to be inconsistent with an evaporation scenario. For example, the speed of the incoming brightening in event 1 is only $20 \mathrm{~km} \mathrm{~s}^{-1}$, whereas the speed for the reflected brightening it is $50 \mathrm{~km} \mathrm{~s}^{-1}$. Furthermore, in event 3 we observed a delay of $20 \mathrm{~s}$ between the arrival of the incoming brightening and the onset of the reflected brightening. These inconsistencies may also mean that these reflected brightenings are rather generated by new reconnection events that occur near these footpoints. Again, co-spatial spectroscopic data are required to substantiate this. It is noteworthy that previous reflection-like phenomena like this seen in large coronal loops in AIA images were interpreted as signatures of slow MHD waves (Kumar et al. 2013; Mandal et al. 2016). Further observations are required to investigate this aspect.

Last, we address the question about the ubiquitousness of propagating brightenings associated with campfires. We recall that by analyzing the same EUI dataset as in this work, Berghmans et al. (2021) reported $\approx 150$ campfires that are more than 5 EUI pixels in size $\left(0.2 \mathrm{Mm}^{2}\right)$ and last for more than $5 \mathrm{EUI}$ frames $(25 \mathrm{~s})$. At the same time, through visual inspections we could only find six events where prominent and systematic propagation signatures are present. We emphasize that in addition to the six cases that we presented here, we visually recognize several other events in our data. Their signal is too ambiguous, however, to establish anything quantitatively. We are therefore currently inclined to conclude that these propagating-type 
localized brightenings are probably not a common phenomenon in the quiet-Sun corona. However, with the availability of data with better spatial (and temporal) resolution from EUI, it may be possible to resolve this disparity. Such data will be available once Solar Orbiter enters its nominal mission phase. Furthermore, it will approach the Sun as close as 0.3 AU during perihelia, which will first be the case early in 2022. This will further improve the spatial resolution.

Acknowledgements. We thank the anonymous referee for helpful comments on the manuscript. This project has received funding from the European Research Council (ERC) under the European Union's Horizon 2020 research and innovation programme (grant agreement No 695075). Solar Orbiter is a mission of international cooperation between ESA and NASA, operated by ESA. The EUI instrument was built by CSL, IAS, MPS, MSSL/UCL, PMOD/WRC, ROB, LCF/IO with funding from the Belgian Federal Science Policy Office (BELSPO/PRODEX PEA 4000112292); the Centre National d'Etudes Spatiales (CNES); the UK Space Agency (UKSA); the Bundesministerium für Wirtschaft und Energie (BMWi) through the Deutsches Zentrum für Luft- und Raumfahrt (DLR); and the Swiss Space Office (SSO).

\section{References}

Alpert, S. E., Tiwari, S. K., Moore, R. L., Winebarger, A. R., \& Savage, S. L. 2016, ApJ, 822, 35

Aschwanden, M. J., Nightingale, R. W., Tarbell, T. D., \& Wolfson, C. J. 2000a, ApJ, 535, 1027

Aschwanden, M. J., Tarbell, T. D., Nightingale, R. W., et al. 2000b, ApJ, 535, 1047

Berghmans, D., \& Clette, F. 1999, Sol. Phys., 186, 207

Berghmans, D., Clette, F., \& Moses, D. 1998, A\&A, 336, 1039

Berghmans, D., Auchère, F., Long, D. M., et al. 2021, A\&A, 656, L4 (SO Cruise Phase SI)

Brosius, J. W., \& Holman, G. D. 2009, ApJ, 692, 492

Brueckner, G. E., \& Bartoe, J. D. F. 1983, ApJ, 272, 329

Chen, Y., Przybylski, D., Peter, H., et al. 2021, A\&A, 656, L7 (SO Cruise Phase SI)
Chitta, L. P., Peter, H., Young, P. R., \& Huang, Y. M. 2017, A\&A, 605, A49 Chitta, L. P., Peter, H., Priest, E. R., \& Solanki, S. K. 2020, A\&A, 644, A130 Chitta, L. P., Peter, H., \& Young, P. R. 2021a, A\&A, 647, A159

Chitta, L. P., Solanki, S. K., Peter, H., et al. 2021b, A\&A, 656, L13 (SO Cruise Phase SI)

De Pontieu, B., Title, A. M., Lemen, J. R., et al. 2014, Sol. Phys., 289, 2733

Dere, K. P., Bartoe, J. D. F., \& Brueckner, G. E. 1989, Sol. Phys., 123, 41

Fisher, G. H., Canfield, R. C., \& McClymont, A. N. 1985, ApJ, 289, 414

Galsgaard, K., Madjarska, M. S., Moreno-Insertis, F., Huang, Z., \& Wiegelmann, T. 2017, A\&A, 606, A46

Harrison, R. A. 1997, Sol. Phys., 175, 467

Hudson, H. S. 1991, Sol. Phys., 133, 357

Innes, D. E., Inhester, B., Axford, W. I., \& Wilhelm, K. 1997, Nature, 386, 811 Innes, D. E., Guo, L. J., Huang, Y. M., \& Bhattacharjee, A. 2015, ApJ, 813, 86 Kobayashi, K., Cirtain, J., Winebarger, A. R., et al. 2014, Sol. Phys., 289, 4393

Krucker, S., Benz, A. O., Bastian, T. S., \& Acton, L. W. 1997, ApJ, 488, 499

Kumar, P., Innes, D. E., \& Inhester, B. 2013, ApJ, 779, L7

Lemen, J. R., Title, A. M., Akin, D. J., et al. 2012, Sol. Phys., 275, 17

Madjarska, M. S. 2019, Liv. Rev. Sol. Phys., 16, 2

Madjarska, M. S., Doyle, J. G., Teriaca, L., \& Banerjee, D. 2003, A\&A, 398, 775

Mandal, S., Yuan, D., Fang, X., et al. 2016, ApJ, 828, 72

Milligan, R. O., Gallagher, P. T., Mathioudakis, M., \& Keenan, F. P. 2006, ApJ, 642, L169

Müller, D., StCyr, O. C., Zouganelis, I., et al. 2020, A\&A, 642, A1

Panesar, N. K., Tiwari, S. K., Berghmans, D., et al. 2021, ApJ, 921, L20

Peter, H., Tian, H., Curdt, W., et al. 2014, Science, 346, 1255726

Rachmeler, L. A., Winebarger, A. R., Savage, S. L., et al. 2019, Sol. Phys., 294, 174

Rochus, P., Auchère, F., Berghmans, D., et al. 2020, A\&A, 642, A8

Solanki, S. K., del Toro Iniesta, J. C., Woch, J., et al. 2020, A\&A, 642, A11

Spice Consortium, (Anderson, M., et al.) 2020, A\&A, 642, A14

Tian, H., Kleint, L., Peter, H., et al. 2014, ApJ, 790, L29

Tiwari, S. K., Panesar, N. K., Moore, R. L., et al. 2019, ApJ, 887, 56

Winebarger, A. R., Walsh, R. W., Moore, R., et al. 2013, ApJ, 771, 21

Young, P. R., Tian, H., Peter, H., et al. 2018, Space Sci. Rev., 214, 120

Zacharias, P., Peter, H., \& Bingert, S. 2011, A\&A, 532, A112

Zhang, Q. M., Chen, P. F., Guo, Y., Fang, C., \& Ding, M. D. 2012, ApJ, 746, 19

Zhukov, A. N., Mierla, M., Auchère, F., et al. 2021, A\&A, 656, A35 (SO Cruise Phase SI) 


\section{Appendix A: Two more cases: Events 5 and 6}
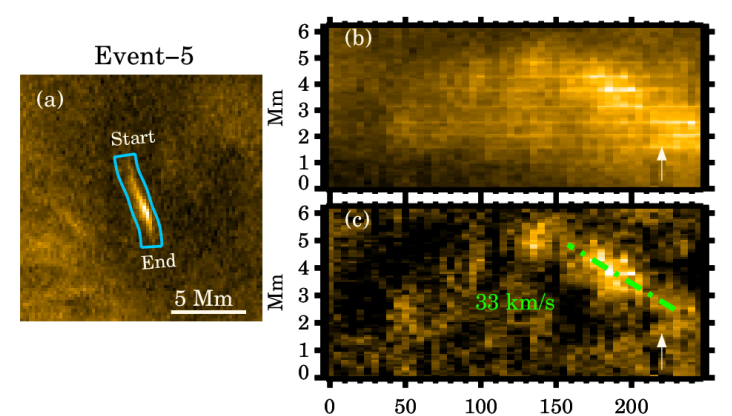

Event-6


Fig. A.1. Overview of event 5 (in panels $a$ and $c$ ) and Event-6 (in panels $d$ and $f$ ). The layout for each event is the same as in Fig. 2a-c. The arrows in panels $\mathrm{b}$ and $\mathrm{c}$ point toward a specific ridge in these maps, as discussed in Appendix A.

The propagation characteristics that we find in event 5 (Fig. A.1a) are generally similar to those of event 3, but with one significant exception. In this case, a small bright patch (of a few pixels) appears to glide over another comparatively larger (extended) bright feature. In the space-time map (Fig A.1b), this appears as a bright arc-like structure that rides on top of a diffuse bright background. A closer look at this X-T map reveals the fragmentary nature of the ridge propagating from "Start" to "End" (i.e., from 0 to $5 \mathrm{Mm}$ ). This happens due to the fact that during this outward motion, the small bright patch appears and disappears multiple times (see also the event movie available online). On the other hand, the return propagation ridge is prominent and continuous. The propagation speed is measured to be $33 \mathrm{~km} \mathrm{~s}^{-1}$ (Fig A.1c). Additionally, we also note a small upward-moving ridge near $t=225 \mathrm{~s}$ (highlighted with the arrows in Fig A.1b and c). This is caused by a new bright feature that appears near what we assume to be the end footpoint and moves upward to meet the return brightening at $t=245 \mathrm{~s}$.

Event 6 (Fig. A.1d) is similar to event 4, but has some differences. In this event, we find a small but isolated bright bloblike feature $\left(0.7 \mathrm{Mm}^{2}\right)$ that propagates from one footpoint to the other, following an arched path. We outline this propagation path with the curved slit shown in Fig. A.1d. The appearance of this blob-like feature is somewhat similar to the dot-like brightenings reported in Tiwari et al. (2019). A blob like this also has been found in a 3D numerical model, but in an active region setting (Zacharias et al. 2011). The bright blob appears close to the end of this observation (starts at $t=200 \mathrm{~s}$ ) and continues to move till the very last frame, where it is seen to merge with an adjacent bright feature. Interestingly, this blob propagates with a significantly higher speed of $118 \mathrm{~km} \mathrm{~s}^{-1}$ ) (Fig. A.1f), which is comparable to the local sound speed (assuming again that the plasma is at a million degrees Kelvin). The speed of this brightening is comparable to the plasma jet speeds reported in Chitta et al. (2021b).
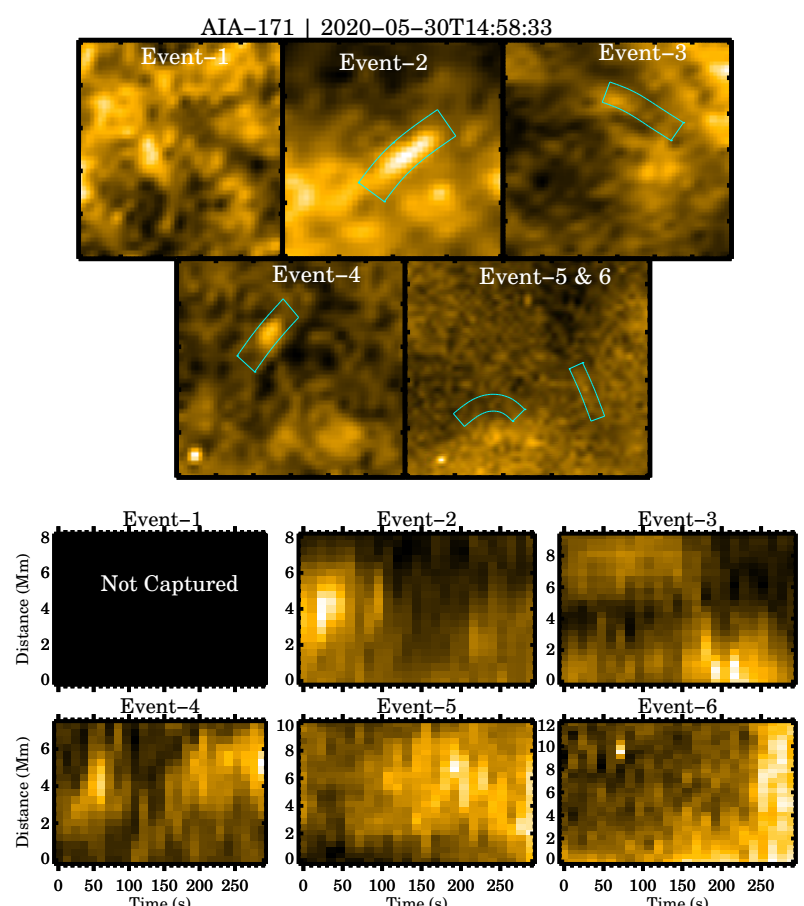

Fig. B.1. AIA view of propagating campfires. The set of five panels at the top presents AIA snapshots of each of our six events. The curved boxes shown in each of these panels mark the locations of the artificial slits we used to derive the temporal evolution in the X-T maps. These X$\mathrm{T}$ maps are shown in the bottom set of panels. Because we were unable to identify event 1 in AIA, we left the corresponding X-T map blank. An animated version of this figure is available online.

\section{Appendix B: AIA view of propagating campfires}

Considering the similarities between the AIA $171 \AA$ and $\mathrm{HRI}_{\mathrm{EUV}}$ $174 \AA$ channels (both passbands capture similar plasma temperatures; Chen et al. 2021, their Fig. 1), it is natural to search for the signatures of these propagating campfires in AIA $171 \AA$ data as well. At this point, we recall that the AIA spatial resolution is only about $900 \mathrm{~km}$, compared to $400 \mathrm{~km}$ in HRI $\mathrm{EUV}_{\mathrm{E}}$ for the observations analyzed here. Furthermore, the AIA EUV image cadence $(12 s)$ is more than twice that of this $\mathrm{HRI}_{\mathrm{EUV}}$ campaign ( $5 \mathrm{~s}$ ). Therefore, these propagating campfires, which typically have widths between 2-6 EUI pixels (and a characteristic propagation timescale of $\approx 40 \mathrm{~s}$ ), are most likely to appear fuzzy and stationary in AIA $171 \AA$ data. As described earlier (in Sect. 2), at the time of this observation (i.e., on 30 May 2020), Solar Orbiter was located at a distance of $0.556 \mathrm{AU}$ with an angle of $31.5^{\circ}$ west in solar longitude from the Earth-Sun line. Thus, the same FOV was also co-observed by AIA. We again refer to Berghmans et al. (2021) for further details. In Fig. B.1 we show the AIA snapshots of each of our EUI events, and we subsequently show corresponding X-T maps that are derived using the cotemporal and coaligned AIA data. As can be seen from these AIA maps, the propagating signatures are largely indistinguishable from the background, and some ridges can even only be identified in hindsight, that is, only after seeing the EUI maps. In case of event 1 , we were only able to see the brightening in two AIA frames, and as a result, were unable to trace its propagation path. From this analysis, we therefore conclude that due to the resolution differences (both spatial and temporal), the signatures of the propagating campfires cannot be identified unambiguously by AIA $171 \AA ̊$ image sequences alone. 


\section{Appendix C: Signal-to-noise ratio}
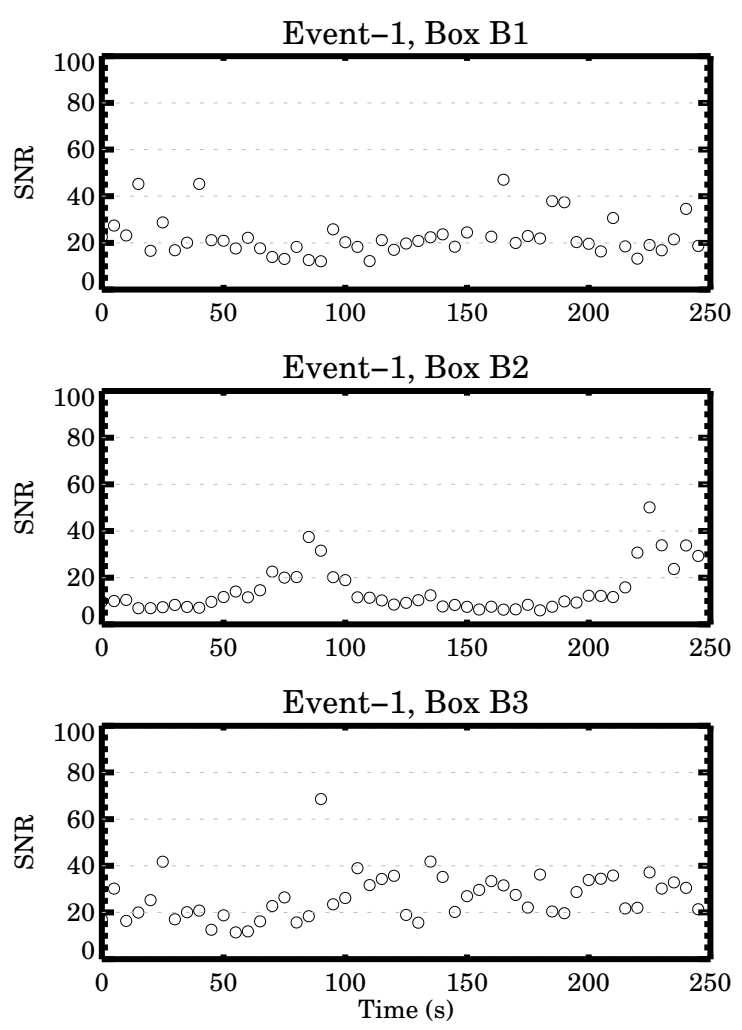

Fig. C.1. Derived $\mathrm{S} / \mathrm{Ns}$ for event 1 . The locations of boxes B1 (top panel), B2 (middle panel), and B3 (bottom panel) are the same as in Fig. 2a.

\section{Event-2}

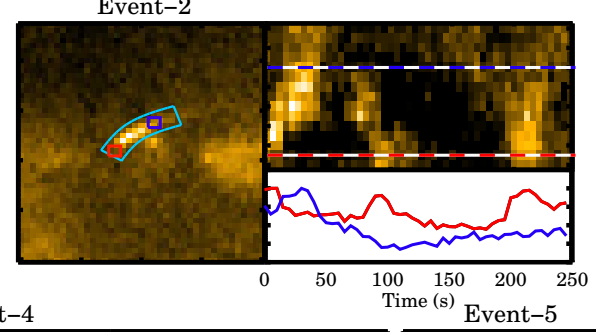

Event-4

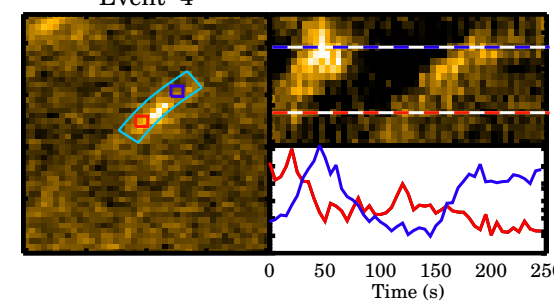

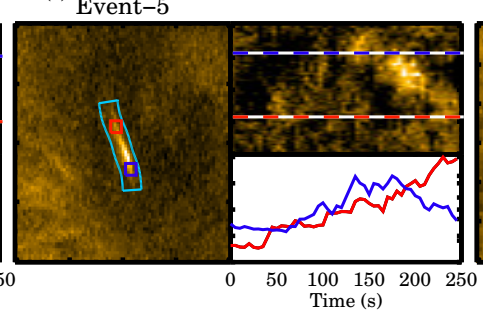

Although the spatial and temporal scales associated with these propagating brightenings are quite close to the instrumental limits, the signal-to-noise ratio $(\mathrm{S} / \mathrm{N})$ of $\mathrm{HRI}_{\mathrm{EUV}}$ images is sufficient to facilitate an unambiguous detection of these events. To obtain a first-order estimate of the noise level in each image, we calculate the mean intensity and the sigma (standard deviation) within a box that encompasses the campfire location. The $\mathrm{S} / \mathrm{N}$ is then defined as the ratio of the mean and sigma, that is, $\mathrm{S} / \mathrm{N}=$ mean/sigma. In Fig. C.1 we show the estimated $\mathrm{S} / \mathrm{Ns}$ for event 1 . These $\mathrm{S} / \mathrm{Ns}$ underline our conclusion that the derived features are real signals.

\section{Appendix D: Light curves of the remaining EUI events}

In Sect. 3.2 we showed the light curves of event 1 (Fig. 2). In Fig. D. 1 we now present the same for the remaining five cases (events 2 to 6). In all of these events, the brightening was either present before the start of the observation (i.e., in the first frame), or the feature was still evolving in the last frame. The complete evolution could therefore not be captured and accordingly, no new information is available through these light curves relative to what is already there in their corresponding X-T maps.

Fig. D.1. EUI light curves of events 2 to 6 . In each of these panels, the curved box (in cyan) highlights the artificial slit, wherein the blue and red boxes mark the locations from which the light curves were derived. The centers of these boxes are also overplotted on top of the associated X-T map as straight horizontal lines. At the bottom of the X-T map, the light curves are shown in red and blue. 